\title{
Estados electrónicos de puntos cuánticos piramidales y cónicos
}

\author{
$\triangle$ John AlEXANDER GIL-CoRrales ${ }^{1}$ \\ ÁlVARo Luis Morales-ARAmbURO ${ }^{1}$ \\ CARlos Alberto DuQue-EcheVERri ${ }^{1}$
}

\section{Resumen}

Los estados electrónicos confinados en un punto cuántico de GaAs, de forma piramidal y cónica, se han investigado a través del enfoque cuasi analítico válido para ángulos pequeños y el método exacto de elementos finitos para incluir todos los ángulos y alturas. Se han reportado los resultados de la energía de confinamiento en función de la forma y el tamaño de ambas estructuras y finalmente se han comparado los valores aproximados con los exactos provenientes del método de elementos finitos.

Palabras clave: Puntos cuánticos piramidales; Puntos cuánticos cónicos; Aproximación cuasianalítica; GaAs; Elementos finitos.

\section{Electronic States of Pyramidal and Conical Quantum Dots}

\section{Abstract}

The electronic states confined in a quantum dot, of GaAs, of pyramidal and conical shape have been investigated through the quasi-analytical approach, valid for small angles, and the finite element method to include all angles and heights. The results of the confinement energy have been reported according to the shape and size of both structures and finally the approximate values have been compared with the exact values obtained from the finite element method.

1 Grupo de Materia Condensada-U. de A., Instituto de Física, Facultad de Ciencias Exactas y Naturales, Universidad de Antioquia U. de A., Medellín, Colombia.

Autor de correspondencia: Gil Corrales, J.A. (John Alexander): Universidad de Antioquia, Calle 70 No. 5221, Medellín, Colombia. Teléfono: (574) 2195630. Correo electrónico: jalexander.gil@udea.edu.co
Historia del artículo:

Artículo recibido: 8-II-2018/ Aprobado: 6-V-2018

Disponible online: 3 de agosto 2018

Discusión abierta hasta abril de 2020 
Keywords: Pyramidal quantum dot; Conical quantum dot; Quasi-analytical aproximation; GaAs; Finite elements.

\section{Estados eletrônicos de pontos quânticos piramidal e cônico}

\section{Resumo}

Os estados eletrônicos confinados em um ponto quântico, de GaAs, de forma piramidal e cônica foram investigados através da abordagem quase-analítica, válida para pequenos ângulos, e o método dos elementos finitos para incluir todos os ângulos e alturas. Os resultados da energia de confinamento foram relatados de acordo com a forma e tamanho de ambas estruturas e, finalmente, os valores aproximados foram comparados com os valores exatos obtidos pelo método dos elementos finitos.

Palavras-chave: Pontos quânticos da piramidal; Pontos quânticos cónicos; Abordagem quase analítica; GaAs; Elementos finitos.

\section{Introducción}

Los puntos cuánticos (QDs) son nano estructuras que cada día toman más importancia en diversas áreas como la medicina, la ingeniería, la industria y las ciencias en general, debido a que sus características particulares de confinamiento del electrón y/o del hueco son de gran importancia para aplicaciones optoelectrónicas y atractivas para el desarrollo de nuevas tecnologías. Debido principalmente al avance experimental en las técnicas de crecimiento de heteroestructuras semiconductoras, se ha vuelto una necesidad la comprensión y el estudio de las propiedades ópticas de los sistemas de confinamiento cuántico, en nano estructuras como pozos cuánticos, hilos cuánticos o puntos cuánticos.

Las nano estructuras de QDs son particularmente importantes en la medicina y la biología. Se han utilizado como sondas fluorescentes para el estudio de los cambios en glicoproteínas del cáncer (Cunha et al. (2018), Andrade et al. (2013) y Pavel et al. (2009)). El ligado de partículas nanométricas con moléculas de bioreconocimiento como péptidos, anticuerpos, etc. (Bailey, Smith y Nie (2004)) permite detectar, por medio del espectro de emi- sión, diferentes anomalías en sistemas biológicos, este fenómeno constituye una de las primeras y más importantes aplicaciones de los QDs a los sistemas de biodetección basados en dispositivos de análisis (Ponnusamy et al. (2007) y referencias anteriores). Además, los puntos cuánticos también están presentes en aplicaciones industriales tales como sensores de temperatura, celdas solares, y aplicaciones optoelectrónicas en moduladores, láseres (Sagadevan y Dakshanamoorthy (2012), Manoj, Hofsass y Vetter (2016), Safeera et al. (2018) y Luhluh (2018)).

Actualmente hay un creciente estudio de las nano estructuras semiconductoras piramidales (PQD). Para estas estructuras es posible obtener los niveles de energía electrónicos (o de hueco) (Duque C.A. et al. (2017) a), hallar los niveles excitonicos y principalmente las transiciones intersubbanda permitidas para obtener las energías de enlace del sistema que son objeto de aplicaciones en el campo del procesamiento cuántico de la información (Hayrapetyan et al. (2016) y Jarlov et al. (2012)). En aplicaciones en dispositivos optoelectrónicos como el diodo emisor de luz, se han realizado estudios experimentales de la electroluminiscencia de puntos cuánticos piramidales en una forma individual 
para potenciales aplicaciones en la fabricación de QD LED de un tamaño micrométrico y se ha llegado a controlar la posición, el número y la densidad de emisores (Baier et al. (2004)).

De igual forma, debido principalmente a sus potenciales aplicaciones en diversos campos son objeto de estudios prominentes las estructuras semiconductoras en forma de cono (Jadupati et al. (2018), Huggenberger et al. (2011) y Pickering et al. (2012)). En este tipo de estructuras se han investigado efectos debidos a la presencia de campos eléctricos y a la posición de alguna impureza interna, generando cambios en las propiedades ópticas del sistema; como por ejemplo, en la rectificación no lineal donde se genera un corrimiento al rojo o al azul dependiendo de la intensidad del campo eléctrico aplicado para un sistema doble de puntos cuánticos cónicos (Bahramiyan (2018), Yamaguchi, Asano, y Noda (2008)). Así mismo, se han realizado análisis en cuanto a la generación de segundo y tercer armónico, para un sistema de punto cuántico cónico, encontrándose una reducción en las energías de transición entre subbandas y un incremento en el solapamiento de los estados cuando se aumentan las dimensiones del sistema (Khordad y Bahramiyan (2014)).

En este trabajo se han llevado a cabo los cálculos exactos de los niveles de energía para los puntos cuánticos piramidal y cónico, por medio de la implementación numérica del método de elementos finitos (FEM), usando el paquete computacional Comsol (COMSOL Multiphysics, v. 5.2a. (2018)). Este método ha sido empleado en trabajos recientes para el estudio de impurezas (Duque C.A. et al. (2017) b), energía de enlace y en el análisis de las propiedades ópticas de puntos cuánticos con forma de lentes para obtener resultados más precisos (Khordad, Bahramiyan y Mohammadi (2016)).

Para este propósito, se han solucionado las ecuaciones de Schrödinger haciendo uso de la aproximación de masa efectiva tanto para el sistema piramidal como para el sistema cónico de GaAs. De igual forma, se han solucionado estas ecuaciones en una aproximación cuasi analítica, utilizando un código fortran desarrollado para este fin, teniendo en cuenta solo ángulos pequeños del vértice para ambos sistemas. Se ha llevado a cabo una comparación entre estos dos métodos, para de esta manera determinar el grado de exactitud de la aproximación cuasi analítica.

\section{Modelo teórico}

El primer sistema a considerar corresponde a un electrón confinado en una estructura piramidal de GaAs con base cuadrada como lo muestra la Figura 1, el potencial de confinamiento es cero en el interior de la estructura e infinito en el exterior de esta.

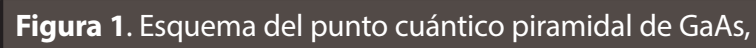
la pirámide tiene una base cuadrada y está caracterizada por la altura $(h)$ y el ángulo del vértice $(\theta)$

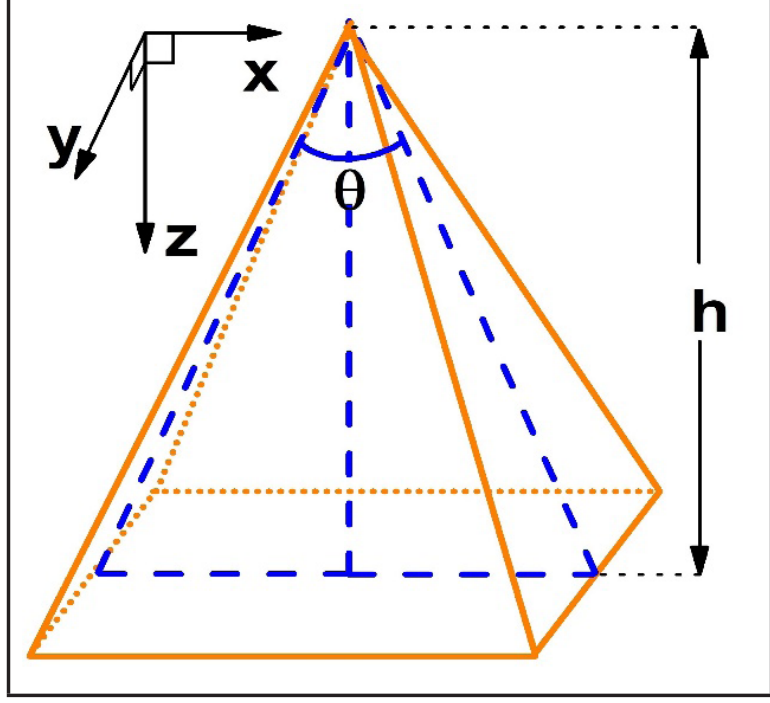

La ecuación de Schrödinger, para un electrón confinado en el PQD, aplicada a este sistema en la aproximación de masa efectiva toma la forma:

$\left[-\frac{\hbar^{2}}{2 m_{*}} \nabla^{2}+V(x, y, z)\right] \Phi(x, y, z)=E \Phi(x, y, z)$

Donde se ha usado la aproximación de masa efectiva en el término $m_{*}$ el cual toma el valor de $m_{*}=0.0665 m_{0}$ (GaAs), $m_{0}$ es la masa del electrón libre, el término $V(x, y, z)$ corresponde al potencial 
de confinamiento de la estructura. El origen de coordenadas se ha tomado en el vértice de la pirámide y las direcciones están definidas como se indica en la Figura 1. Teniendo en cuenta lo anterior, las condiciones de frontera para el problema toman la forma: $z=a x$ para $x>0, z=-a x$ para $x<0, z=a y$ para $y>0 \mathrm{y} z=-a y$ para $y<0$, para $0 \leq z \leq h$, donde $a=1 / \tan (\theta / 2)$.

Con estas condiciones de frontera, el problema no puede ser solucionado analíticamente, por esta razón se propone una solución cuasianalítica (tomando como base el trabajo de Lozovski V. y Piatnytsia V. (2011)) que es válida para ángulos apicales no superiores a $90^{\circ}$. Es conveniente realizar una transformación de coordenadas de la forma: $\alpha=x / z$, $\beta=y / z$ y $\gamma=z$, que conllevan a las transformaciones inversas: $x=a \gamma, y=\beta \gamma \mathrm{y} z=\gamma$, el dominio para estas nuevas variables se obtiene por medio de las condiciones de frontera iniciales y está dado por: $-1 / a \leq \alpha, \beta \leq 1 / a$ y $0 \leq \gamma \leq h$. Recordando que la estructura no posee ángulos demasiado grandes debe cumplirse que $\alpha, \beta \ll 1$. Con estas consideraciones, la Ecuación (1) puede desarrollarse en el nuevo sistema de coordenadas para obtener un conjunto de tres ecuaciones no acopladas,

$$
\begin{gathered}
{\left[\frac{d^{2}}{d \alpha^{2}}+\alpha \frac{d}{d \alpha}\right] \phi(\alpha)=k_{\alpha}^{2} \phi(\alpha)} \\
{\left[\frac{d^{2}}{d \beta^{2}}+\beta \frac{d}{d \beta}\right] \varphi(\beta)=-k_{\beta}^{2} \varphi(\beta)} \\
\frac{1}{\gamma^{2}} \frac{d}{d \gamma}\left(\gamma^{2} \frac{d \xi(\gamma)}{d \gamma}\right)=\left(\frac{k_{\alpha}^{2}+k_{\beta}^{2}}{\gamma^{2}}-k_{l}^{2}\right) \xi(\gamma)
\end{gathered}
$$

Los autovalores de la energía del sistema pueden ser obtenidos como: $E_{l}=k_{l}^{2} \hbar^{2} / 2 m_{*}$. Las Ecuaciones (2) y (3) pueden simplificarse teniendo en cuenta que el sistema solo trata ángulos pequeños $(\alpha, \beta \ll 1)$ y por lo tanto se cumplirá que $d^{2} \phi(\alpha) /$ $d \alpha^{2}>>\alpha(d \phi(\alpha) / d \alpha)$ y $d^{2} \varphi(\beta) / d \beta^{2}>>\beta(d \varphi(\beta) / d \beta)$, despreciando los términos correspondientes en ambas ecuaciones se obtienen las autofunciones que corresponden a una solución cuasianalítica del problema

$$
\begin{gathered}
\phi_{n} \sim \sin \left(\frac{k_{\alpha n}}{a}\right) \cos \left(k_{\alpha n} \alpha\right)+\cos \left(\frac{k_{\alpha n}}{a}\right) \sin \left(k_{\alpha n} \alpha\right) \\
k_{\alpha n}=\frac{n \pi a}{2}, \quad n=1,2,3, \ldots
\end{gathered}
$$

y

$$
\begin{gathered}
\phi_{m} \sim \sin \left(\frac{k_{\beta m}}{a}\right) \cos \left(k_{\beta m} \beta\right)+\cos \left(\frac{k_{\beta m}}{a}\right) \sin \left(k_{\beta m} \beta\right) \\
k_{\beta m}=\frac{m \pi a}{2}, \quad m=1,2,3, \ldots
\end{gathered}
$$

Es sencillo demostrar que la Ecuación (4) posee soluciones proporcionales a las funciones de Bessel de la forma

$$
\xi(\gamma) \sim \frac{1}{\sqrt{\gamma}} J_{\delta}\left(k_{l} \gamma\right)
$$

Donde $\delta=\sqrt{k_{a n}^{2}+k_{\beta m}^{2}+0.25}$. Se ha tenido en cuenta el confinamiento infinito de la estructura lo que conlleva a que la función de onda se anule en todas las fronteras del punto cuántico. Adicionalmente, es posible escribir la función de onda completa del sistema, en la aproximación cuasi analítica, en las variables originales $x, y, z$ como

$$
\begin{gathered}
\phi_{n, m, l}(x, y, z)=\frac{N}{\sqrt{Z}} J_{\delta}\left(k_{,},\right) \times \\
{\left[\sin \left(\frac{k_{\alpha n}}{a}\right) \cos \left(\frac{k_{\alpha n} X}{Z}\right)+\cos \left(\frac{k_{\alpha n}}{a}\right) \sin \left(\frac{k_{\alpha n} X}{Z}\right)\right] \times} \\
{\left[\sin \left(\frac{k_{\beta m}}{a}\right) \cos \left(\frac{k_{\beta m} y}{Z}\right)+\cos \left(\frac{k_{\beta m}}{a}\right) \sin \left(\frac{k_{\beta m} y}{Z}\right)\right]}
\end{gathered}
$$

Donde $N$ es la constante de normalización del sistema y $k_{l} h$ corresponde al l-ésimo cero de la función de Bessel, las constantes $n, m$ y $l$ son los números cuánticos del problema.

El segundo sistema considerado es el de un electrón confinado es una estructura de GaAs de forma cónica, como lo muestra la Figura 2. 
Figura 2. Esquema del punto cuántico cónico de GaAs, el cual está caracterizado por la altura ( $h$ ) y el ángulo del vértice $(\theta)$. El potencial de confinamiento es cero en el interior de la estructura e infinito en el exterior.

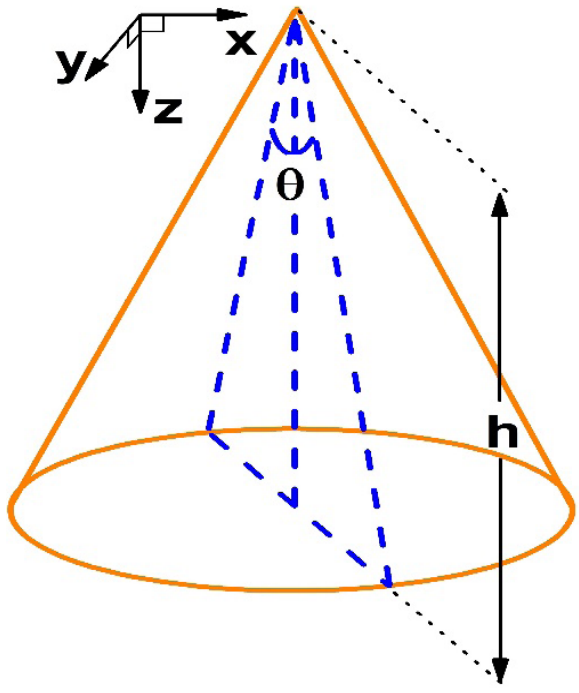

La ecuación de autovalores del sistema es nuevamente la Ecuación (1), donde solo se presentan variaciones en la forma del potencial de confinamiento, teniendo en cuenta el mismo valor para la constante $a$ en las ecuaciones anteriores para la pirámide, el dominio para el sistema cónico está dado por $z=a \sqrt{x^{2}+y^{2}}$ donde $0 \leq z \leq h$. Es conveniente nuevamente realizar una transformación de coordenadas de la forma $x=\alpha \gamma, \gamma=\beta \gamma \mathrm{y} z=\gamma$, posteriormente y aprovechando la simetría de la estructura cónica es posible reescribir el problema por medio de coordenadas cilíndricas como: $\alpha=\eta \cos (\sigma), \beta=$ $\eta \sin (\sigma)$ con $0 \leq \gamma \leq h$ y $0 \leq \eta \leq 1 / a$. Considerando nuevamente ángulos del vértice inferiores a $90^{\circ}$, es posible luego de un desarrollo sencillo de la Ecuación (1) despreciar ciertos términos en la expresión del laplaciano, más concretamente términos con dependencia cuadrática de $\eta$, a partir de este punto se puede escribir la función de onda completa del sistema como un producto de funciones individuales en cada una de las nuevas variables,

$$
\Phi(\sigma, \eta, \gamma)=\phi(\sigma) \varphi(\eta) \xi(\gamma)
$$

Teniendo en cuenta este resultado y las aproximaciones hechas en el párrafo anterior, es posible obtener un sistema de tres ecuaciones desacopladas para cada una de las funciones dependientes de cada coordenada,

$$
\begin{gathered}
\frac{d^{2} \phi(\sigma)}{d \sigma^{2}}=-m^{2} \phi(\sigma) \\
{\left[\frac{d^{2}}{d \eta^{2}}+\frac{1}{\eta} \frac{d}{d \eta}\right] \varphi(\eta)=\left(\frac{m^{2}}{\eta^{2}}-k_{a}^{2}\right) \varphi(\eta)} \\
\frac{1}{\gamma^{2}} \frac{d}{d \gamma}\left(\gamma^{2} \frac{d \xi(\gamma)}{d \gamma}\right)=\left(\frac{m^{2}}{\gamma^{2}}-k_{b}^{2}\right) \xi(\gamma)
\end{gathered}
$$

La Ecuación (10) genera una autofunción,

$$
\phi_{n}(\sigma)=N_{\sigma} e^{i m \sigma}
$$

Donde $N_{\sigma}$ es la constante de normalización correspondiente a la coordenada $\sigma, i=\sqrt{-1}$ es la unidad imaginaria y $m=0, \pm 1, \pm 2, \ldots$ Es sencillo demostrar que las autofunciones de las Ecuaciones (11) y (12) son proporcionales a las funciones de Bessel y tienen la forma:

$$
\varphi_{m}(\eta)=N_{\eta} J_{m}\left(k_{a} \eta\right)
$$

Donde $N_{\eta}$ es la constante de normalización correspondiente a la coordenada $\eta$,

$$
\xi(\gamma) \sim \frac{N_{\gamma}}{\sqrt{\gamma}} J_{\delta}\left(k_{b} \gamma\right)
$$

Con $\delta=\sqrt{k_{a}^{2}+0.25}, N_{\gamma}$ es la constante de normalización correspondiente a la coordenada $\gamma$, recordando que el potencial exterior es infinito, luego la función de onda debe anularse en la frontera de la estructura, lo cual conlleva a las dos ecuaciones $J_{m}\left(k_{a}^{m i} / a\right)=0, \mathrm{y} J_{\delta}\left(k_{b}^{m i j} h\right)=0$ cuyas soluciones dan los valores de $k_{a}^{m i}$ y $k_{b}^{m i j}$ para cada $i, j$ donde $i, j=1,2,3$, ... son los ceros de cada una de estas dos ecuaciones.

Nuevamente es posible escribir la función de onda del sistema completo en la aproximación cuasianalítica en las variables originales $x, y, z$

$$
\begin{gathered}
\Phi^{m i j}(x, y, z)=\frac{N}{\sqrt{z}} J_{m}\left(k_{a}^{m i} \frac{\sqrt{x^{2}+y^{2}}}{z}\right) \times \\
J_{\delta}\left(k_{b}^{m i j} z\right) e^{i m \tan ^{-1}(y / x)}
\end{gathered}
$$


Donde $N$ es la constante de normalización y $m$, $i, j$ son los números cuánticos del sistema. Finalmente, los autovalores de energía pueden ser obtenidos ahora como:

$$
E_{m i j}=\frac{\hbar^{2}\left(k_{b}^{m i j}\right)^{2}}{2 m_{*}}
$$

Donde $m_{*}$ es la masa efectiva electrónica para el sistema cónico de GaAs.

En la Figura 3 se observa la malla usada para resolver la ecuación de Schrödinger para el sistema piramidal y el sistema cónico, por medio de la implementación del método de elementos finitos.

Los elementos interiores, que conforman la malla, para cada una de las estructuras son de tipo tetraedro, el tamaño y la cantidad de estos se ajusta a medida que se realizan variaciones en el tamaño de las estructuras. Como ilustración, para una altura de $15 \mathrm{~nm}$ y un ángulo del vértice de $30^{\circ}$, la malla es construida para la pirámide con 133066 elementos tetraédricos y con 6590 elementos de contorno que corresponden a elementos triangulares como se observa en la Figura 3. Para las mismas dimensiones de altura y ángulo apical, la malla correspondiente para el cono contiene 108634 elementos tetraédricos y 6178 elementos triangulares para el contorno.

Figura 3. Malla usada para la implementacion del método de elementos finitos para el sistema piramidal y el sistema cónico.

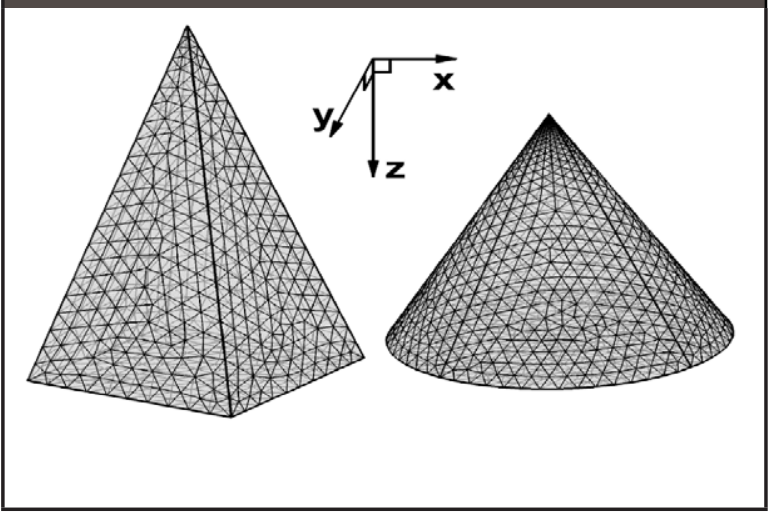

Se han impuesto condiciones de frontera de Dirichlet tanto para el cono como para la pirámide, es decir, que las funciones de onda se anulen en los bordes.

\section{Resultados y discusión}

Los parámetros para GaAS usados para el desarrollo numérico (método de elementos finitos y aproximación cuasi analítica) tanto de la pirámide como del cono fueron los siguientes: constante dieléctrica $\varepsilon=12.35$, permitividad del vacio $\epsilon_{0}=$ $8.85 \times 10^{-12} \mathrm{~F} / \mathrm{m}$, la masa efectiva del electrón $m_{*}=$ $0.0665 m_{0}, m_{0}$ es la masa del electrón libre. En la Figura 4 se presentan los primeros seis niveles de energía de un electrón confinado en un punto cuántico piramidal (a) y cónico (b) como función del ángulo del vértice. Los resultados presentados son exactos y se han obtenido por medio de la implementación del método de elementos finitos, manteniendo fija la altura de ambas estructuras en $h=15 \mathrm{~nm}$.

Es posible observar un claro comportamiento decreciente de las curvas de energía para ángulos grandes, esto se debe a que el volumen de la estructura es cada vez mayor y por lo tanto se presenta una pérdida de confinamiento electrónico. Este comportamiento decreciente es un poco más significativo en el sistema piramidal que en el sistema cónico y se debe principalmente a que el volumen del sistema piramidal aumenta más rápidamente que el volumen del sistema cónico cuando se hace la variación del ángulo del vértice, esto puede verse claramente en los recuadros de cada figura. Los números que se presentan junto a cada una de las curvas indica el orden de degeneración de cada uno de los estados. Es de resaltar que debido a la forma cónica del punto cuántico se presenta un mayor confinamiento para un mismo ángulo del vértice que para el punto cuántico piramidal, esto se observa claramente ya que las curvas del cono están desplazadas hacia energías superiores con respecto a las curvas piramidales. Como es de esperarse, debido a que el sistema cónico presenta un mayor orden de simetría, luego se debe esperar un mayor número de estados degenerados por simetría que para el sistema piramidal. 
Figura 4. Primeros seis niveles de energía de un electrón confinado en un punto cuántico piramidal (a) y cónico (b), como función del ángulo apical. En los recuadros se muestra la variación respectiva del volumen con el ángulo y la altura para ambos sistemas. Funciones de onda de los 3 primeros estados excitados para el sistema piramidal. El panel (c) corresponde a $h=15 \mathrm{~nm}$ y $\theta=40^{\circ}$, El panel (d) a $h=15 \mathrm{~nm}$ y $\theta=50^{\circ}$, la figura de izquierda a derecha corresponde a las proyecciones en los planos $x y, x z$ y yz respectivamente. Los planos se han tomado en el centro de gravedad de la estructura.
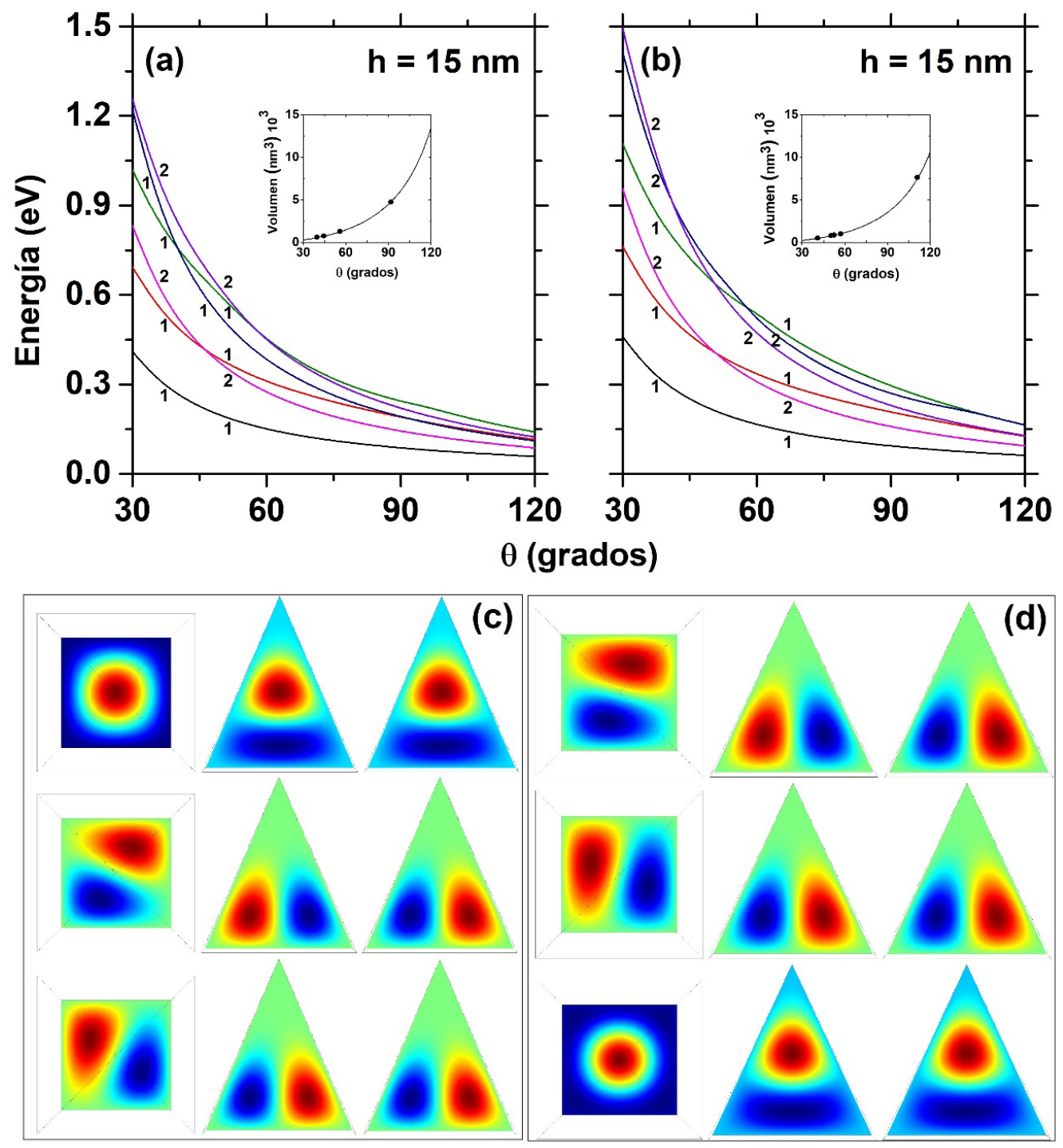

Tanto para la pirámide como para el cono se presentan cruces de los niveles que corresponden a degeneración accidental. Para la pirámide, en $\theta=$ $46^{\circ}$ los dos primeros estados excitados con números cuánticos $(1,1,2)$ y $(2,1,1)$, este ultimo presenta una doble degeneración con el estado $(1,2,1)$. Hay que resaltar que este cruce se presenta entre estados tipo $p$. De forma similar para el sistema cónico se presenta el cruce para $\theta=51^{\circ}$ entre los estados $(0,1,2)$ y $(1,1,1)$, nuevamente ambos estados corresponden a estados tipo $p$. Para el sistema piramidal igualmente se presenta un cruce de los estados tipo $d(1,1,3)$ y $(2,2,1)$ en aproximadamente $\theta=40^{\circ}$, para el sistema cónico, este cruce se da en alrededor de $\theta=50^{\circ}$ de forma similar para estados tipo $d$. En los recuadros de la Figura 4 se ha presentado la 
variación del volumen de cada sistema a medida que se aumenta el ángulo de cada estructura, los puntos negros ubicados sobre la curva indican los puntos donde se presentan cruces en los estados electrónicos y por lo tanto corresponden a puntos de degeneración accidental del sistema. Este tipo de degeneración resulta de la forma funcional del potencial que se considera o de características particulares del sistema y son evidenciados experimentalmente en un acuerdo con los resultados teóricos (Norris D. J. y Bawendi M. G. 1996). Hay que tener en cuenta que el espectro de energía presenta una evolución fuerte con el régimen de confinamiento del sistema. Note que el orden de los estados depende directamente del ángulo del vértice de la estructura. Los restantes estados excitados igualmente presentan cruces que son importantes para un estudio detallado de las propiedades ópticas de los sistemas.

En la Figura 4 (c) y (d) se observan las funciones de onda del sistema piramidal correspondientes a los tres primeros estados excitados del sistema. Las Figuras (c) y (d) conciernen a ángulos de $\theta=40^{\circ}$ y $\theta$ $=50^{\circ}$ respectivamente, manteniendo la misma altura de $h=15 \mathrm{~nm}$. Como lo indica el panel (a), el tercer y cuarto estado excitado corresponde a un estado doblemente degenerado. Se puede apreciar cómo se presenta un intercambio de los estados cuando se aumenta el sistema en los ángulos señalados. El primer estado excitado pasa a ocupar la posición del tercer estado excitado, este comportamiento está en perfecto acuerdo con la Figura 4 donde se puede apreciar un cruce entre estos estados para un ángulo de $\theta=$ $46^{\circ}$ para el sistema piramidal. El sistema cónico presenta un comportamiento similar, presentándose el intercambio de los estados en este caso para $\theta=51^{\circ}$.

En la Figura 5 se observan los primeros seis niveles de energía para un punto cuántico piramidal (a) y cónico (b) como función de la altura de la estructura, manteniendo fijo el ángulo del vértice en $\theta$ $=60^{\circ}$. Los resultados son obtenidos al igual que en la Figura 4 por medio del método numérico de elementos finitos usando las mallas presentadas en la Figura 3. Nuevamente se presenta un comportamiento de- creciente de los niveles energéticos a medida que se aumenta la altura de las estructuras. Note que en la Figura 5a, el estado de mayor energía es doblemente degenerado. Este decaimiento es más pronunciado en el caso mostrado en la Figura 4, esto se debe a que el volumen de ambos puntos cuánticos aumenta más rápidamente con la variación del ángulo que con la variación de la altura, lo cual genera una pérdida de confinamiento mayor cuando se varia el ángulo y por lo tanto las energías tienden a bajar más rápidamente en la Figura 4 que en la Figura 5. Esto puede verse claramente comparando los recuadros de las Figuras 4 y 5. Es importante resaltar que para este caso no se presenta degeneración accidental para ninguno de los estados presentados, sin embargo, sí se dan estados excitados degenerados por simetría. Al igual que en el caso de la altura fija, las curvas correspondientes al cono presentan un desplazamiento hacia energías superiores con respecto a las mismas para la pirámide, debido a que la forma cónica presenta un confinamiento mayor para una misma altura manteniendo el ángulo apical fijo.

Los resultados obtenidos en las Figuras 4 y $\mathbf{5}$ están en total acuerdo con los resultados reportados en la literatura que indican un comportamiento decreciente de los niveles de energía con el aumento del volumen de las estructuras, igualmente se han obtenido degeneraciones accidentales para algunos niveles excitados cuando se varia el ángulo del vértice que están de acuerdo con trabajos anteriores.

En la Figura 6 se presentan los primeros seis niveles de energía de un punto cuántico piramidal (a) y cónico (b) como función del ángulo del vértice, manteniendo el volumen de la estructura fijo para $h$ $=15 \mathrm{~nm}$ y $\theta=120^{\circ}$. Los niveles de energía tanto para el cono como para la pirámide presentan una fuerte dependencia con el ángulo del vértice de la estructura. Igualmente se dan degeneraciones para ciertos ángulos particulares, para $\theta=46^{\circ}$ y $\theta=51^{\circ}$ se degeneran los dos primeros estados excitados para la pirámide y el cono respectivamente, lo mismo ocurre para el tercer y cuarto estados excitados que se degeneran para cada estructura en $\theta=40^{\circ}$ y para $\theta=50^{\circ}$. 
Hay que resaltar que todos los estados presentan una tendencia a aumentar su energía a medida que se aumenta el ángulo del vértice, esto es una consecuencia únicamente debida al cambio en la forma del punto cuántico ya que el volumen permanece constante.
Este aumento de energía se debe a que, al aumentar el ángulo de la estructura, debe disminuir la altura de esta para mantener así el volumen fijo, luego el confinamiento es mucho más significativo con la base de la estructura que con las paredes de esta.

Figura 5. Primeros seis niveles de energía de un electrón confinado en un punto cuántico piramidal (a) y cónico (b), como función de la altura. En los recuadros se muestra la variación respectiva del volumen con el ángulo y la altura para ambos sistemas.

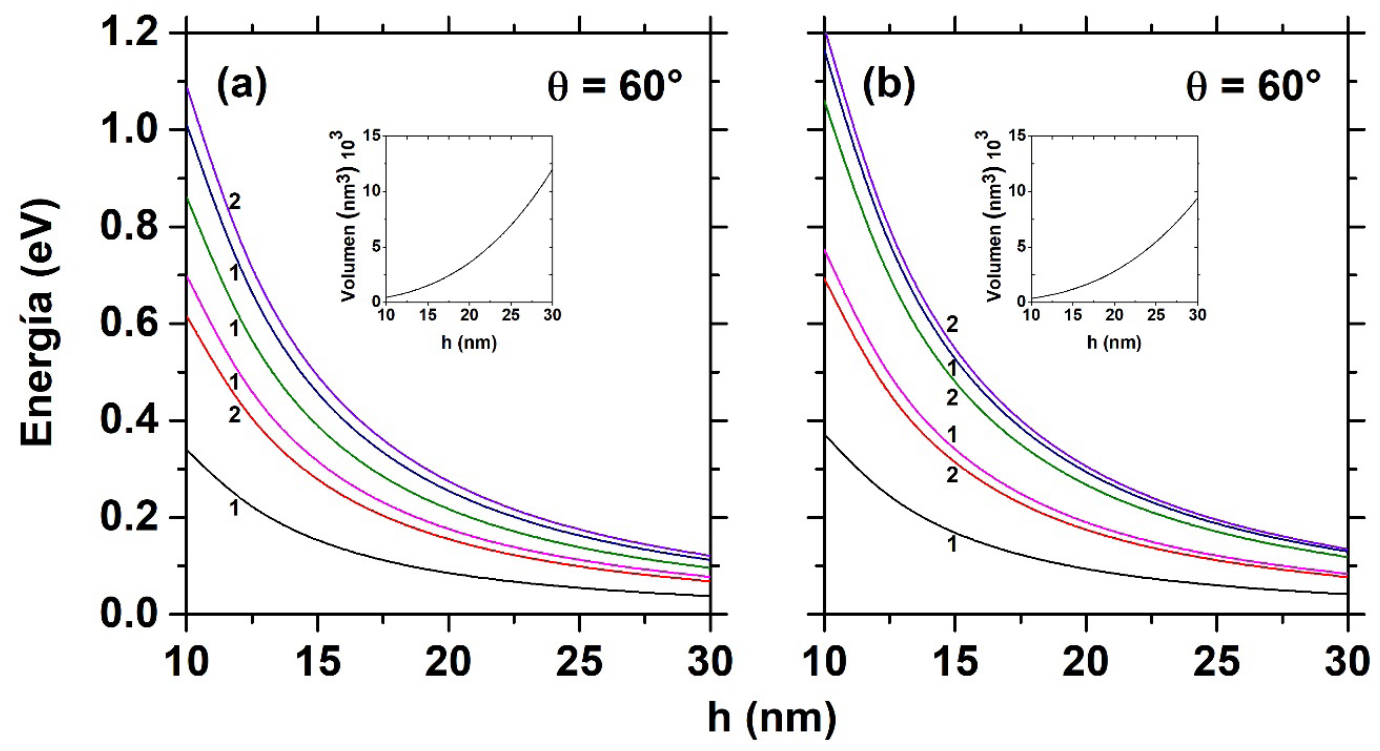

Figura 6. Primeros seis niveles de energía de un electrón confinado en un punto cuántico piramidal (a) y cónico (b), como función del ángulo apical. Los cálculos son exactos y realizados manteniendo fijo el volumen de la estructura para $\theta=120^{\circ}$ y $h=15 \mathrm{~nm}$.

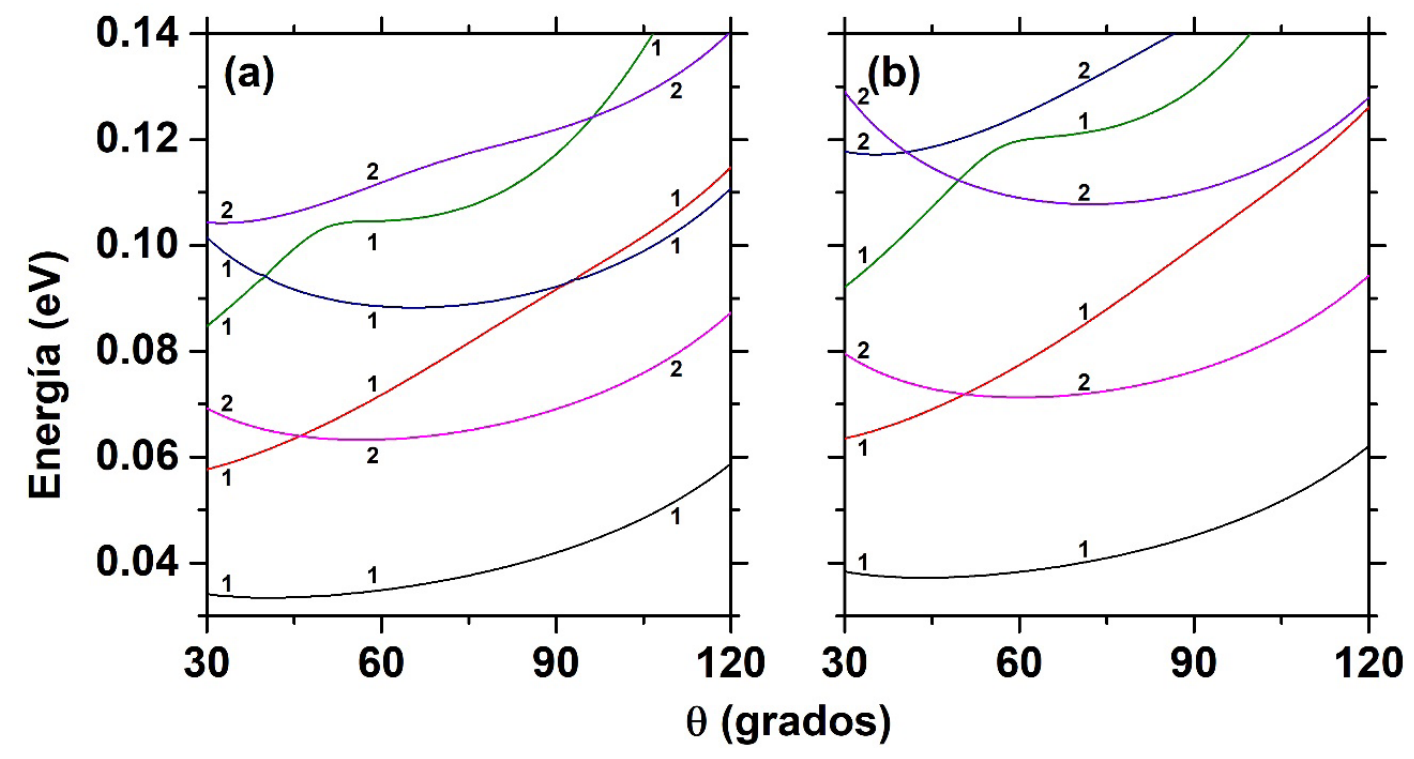


Figura 7. Primeros seis niveles de energía de un electrón confinado en un punto cuántico piramidal (a) y cónico (b), como función de la altura. Los cálculos son exactos y realizados manteniendo fijo el volumen de la estructura para $\theta=120^{\circ}$ y $h=15 \mathrm{~nm}$.

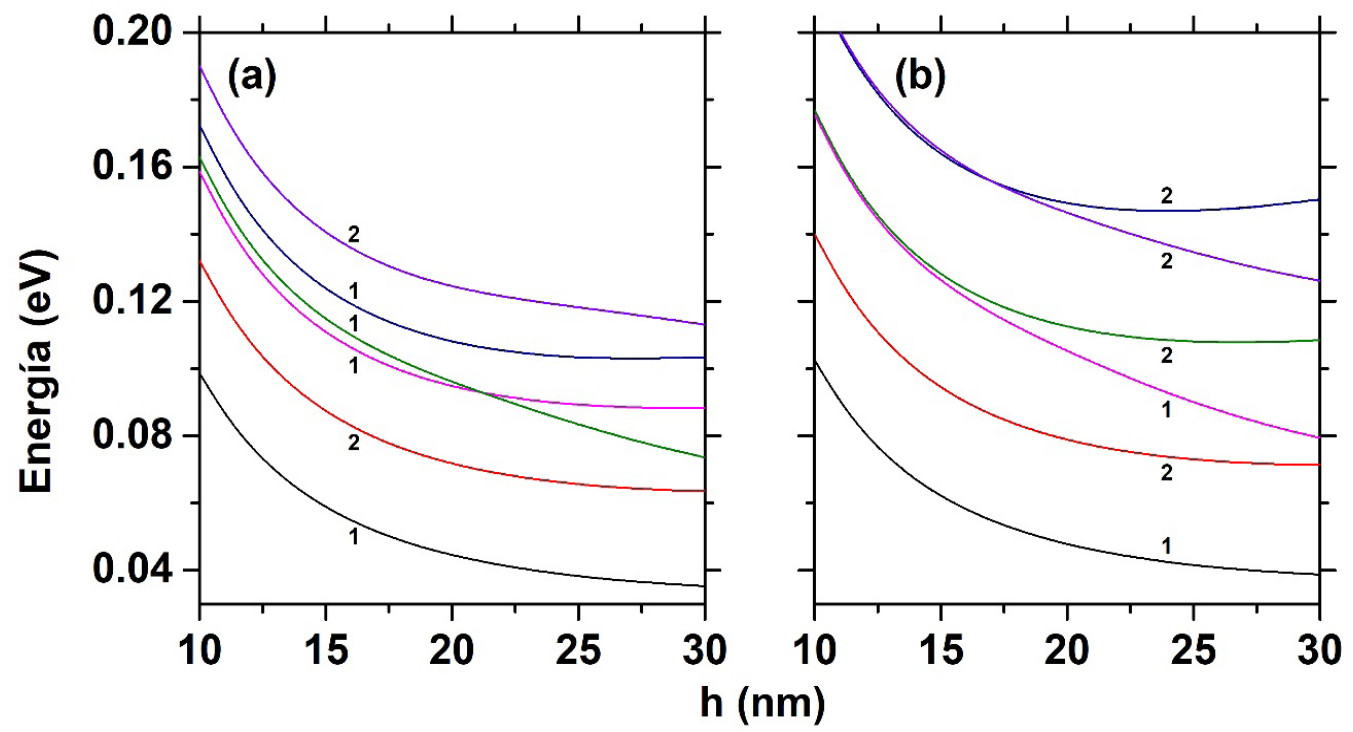

Figura 8. Comparación de los primeros seis niveles de energía para el punto cuántico piramidal, como función del ángulo del vértice, manteniendo el volumen constante $\operatorname{con} h=15 \mathrm{~nm}$ y $\theta=120^{\circ}$

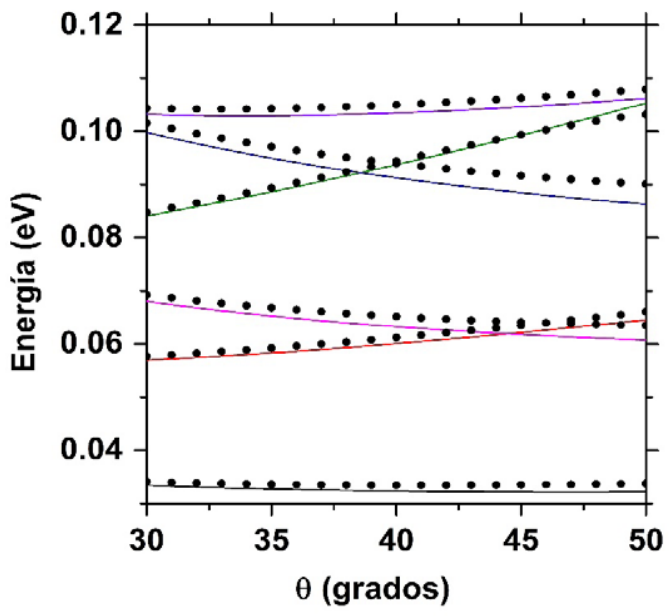

En la Figura 7 se presentan igualmente los primeros seis niveles de energía para los dos sistemas estudiados, piramidal (a) y cónico (b). En este caso se realizan variaciones en la altura de las estructuras, manteniendo el mismo volumen fijo que se ha usado en la Figura 6, con $h=15 \mathrm{~nm}$ y $\theta=120^{\circ}$. Es de resaltar que los niveles de energía presentan un comportamiento creciente para ángulos muy grandes en la Figura 6, mientras que en esta figura los niveles de energía son todos decrecientes, lo cual es consecuencia de que el confinamiento en la coordenada $z$ es mucho más significativo que en el plano $x y$.

En la Figura 8 se presenta una comparación de los primeros seis niveles de energía para el punto cuántico piramidal como función del ángulo del vértice, manteniendo fijo el volumen de la estructura con los parámetros $h=15 \mathrm{~nm}$ y $\theta=120^{\circ}$. Las líneas continuas corresponden a los resultados cuasi analíticos y los puntos corresponden a los resultados exactos. Para ángulos pequeños la aproximación se cumple en gran medida principalmente para estados de baja energía (a pesar que se ha tomado como punto de partida para el volumen fijo un ángulo muy superior a lo aceptado en los cálculos aproximados). Hay que resaltar que el sistema se encuentra a un volumen suficientemente grande y por lo tanto en un régimen de bajas energías (en el rango de $0.03 \mathrm{eV}$ a $0.12 \mathrm{eV}$ ), esto puede verse revisando la Figura 4 y el recuadro del volumen como función del ángulo del vértice, donde se puede apreciar que se está en el rango máximo del volumen y mínimo en la energía. 
Figura 9. Comparación de los primeros seis niveles de energía para el punto cuántico piramidal (a) y cónico (b), como función del ángulo del vértice, para $h=15 \mathrm{~nm}$. Las líneas continuas corresponden a los resultados cuasi analíticos, los puntos corresponden a los resultados exactos. La flecha indica el orden de los estados caracterizados por sus números cuánticos de acuerdo a los cálculos analíticos.

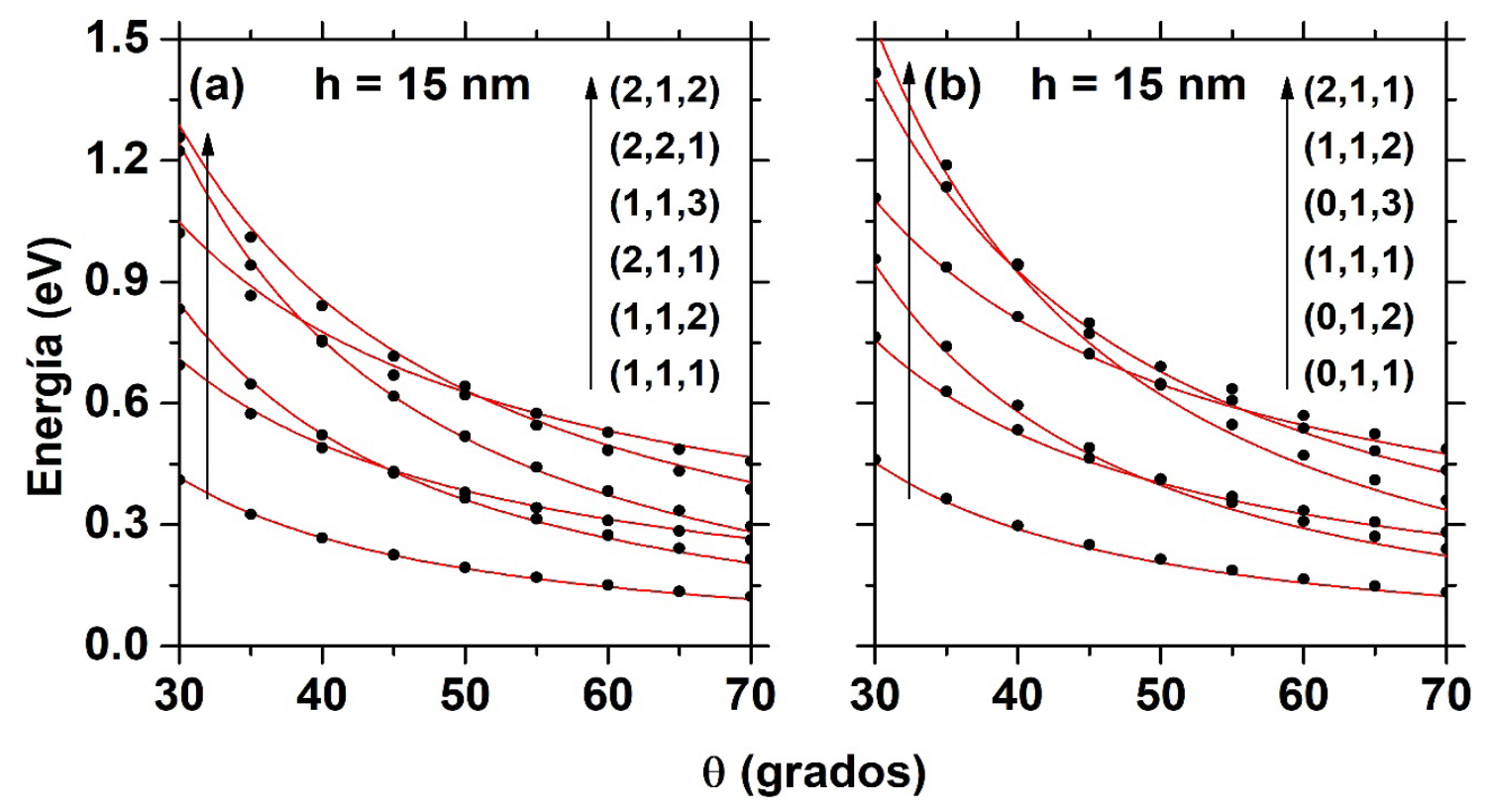

Figura 10. Comparación de los primeros seis niveles de energía para el punto cuántico piramidal (a) y cónico (b), como función de la altura, para $\theta=60^{\circ}$. Las líneas continuas corresponden a los resultados cuasi analíticos, los puntos corresponden a los resultados exactos. La flecha indica el orden de los estados caracterizados por sus números cuánticos de acuerdo con los cálculos analíticos.
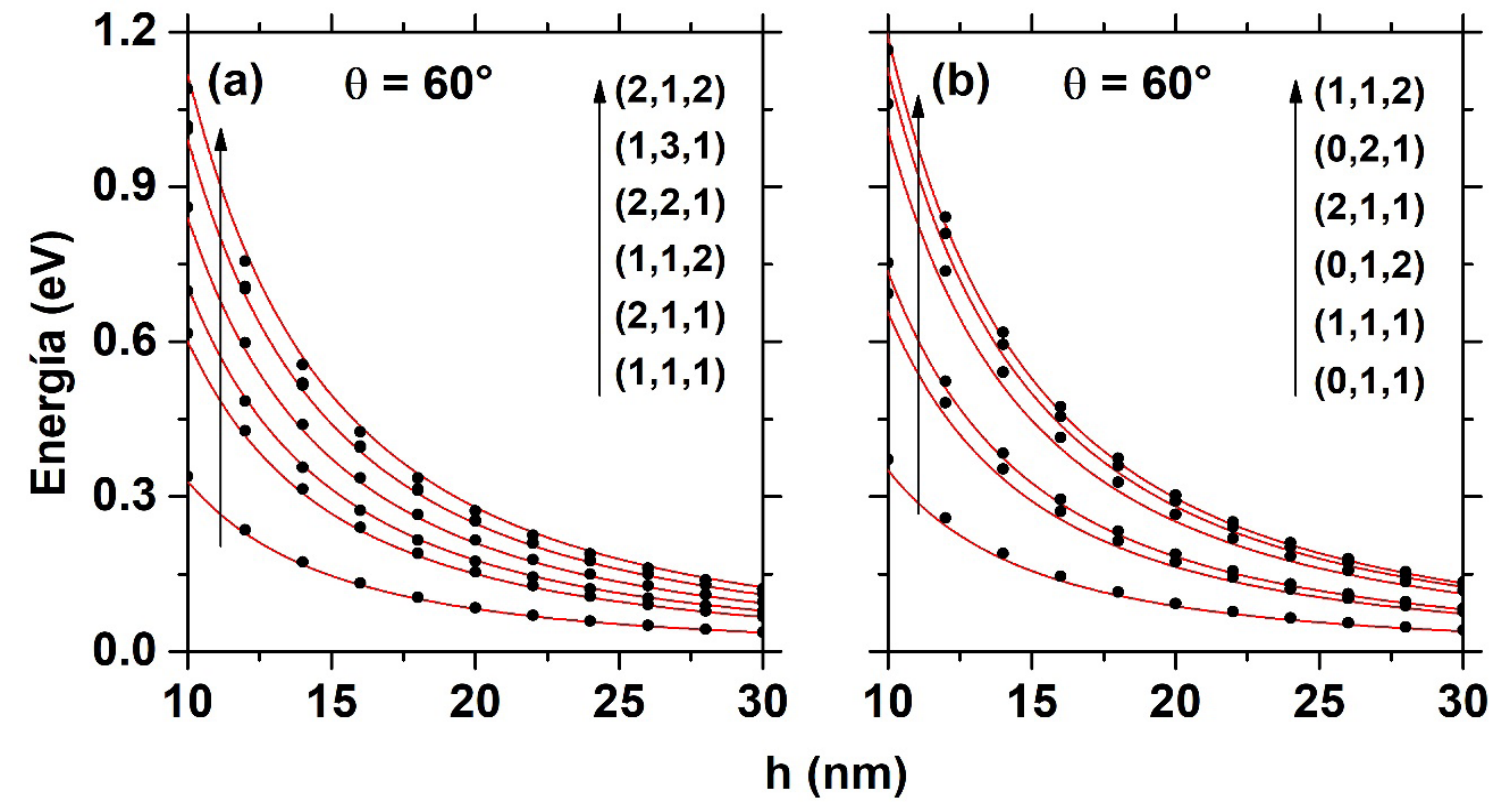
En la Figura 9 se presenta una comparación de los primeros seis niveles de energía para el punto cuántico piramidal (a) y cónico (b) como función del ángulo del vértice, manteniendo la altura fija en $h=15 \mathrm{~nm}$. Las líneas continuas corresponden a los resultados cuasi analíticos, es decir, son aproximados y son válidos para ángulos pequeños, los puntos corresponden a los resultados numéricos obtenidos por medio del método de elementos finitos. La flecha en la figura indica el orden de cada uno de los estados. Los puntos caen de forma precisa sobre las curvas, especialmente para los estados más bajos, los cuales prácticamente no se ven afectados por el aumento en el ángulo del vértice tanto para la pirámide como para el cono, esto indica que la aproximación cuasi analítica usada para resolver el sistema de Ecuaciones (2), (3) y (4) concuerda en gran medida con los resultados exactos y en efecto es una muy buena aproximación para resolver este sistema para ángulos pequeños. Los estados que se alejan más de los resultados exactos son los de energía superior, sin embargo, presentan un muy buen comportamiento en el rango de ángulos en los cuales es válida la aproximación. Es importante notar que los números cuánticos que se presentan para algunos estados excitados pueden variar ya que se presentan degeneraciones en el sistema y por lo tanto existen configuraciones distintas con igual energía, igualmente, el sistema cónico puede tomar el cero para el primer número cuántico, mientras que el sistema piramidal solo puede empezar en uno.

En la Figura 10 se presenta una comparación de los primeros seis niveles de energía para el punto cuántico piramidal como función de la altura, manteniendo el ángulo del vértice fijo en $\theta=60^{\circ}$. Nuevamente las líneas continuas corresponden a los resultados cuasi analíticos, mientras que los puntos presentan los resultados usando el método de elementos finitos. Para el punto cuántico piramidal prácticamente no se dan variaciones con respecto a ambas soluciones para todos los estados a medida que se aumenta la altura de la estructura, esto se debe a que la aproximación cuasi analítica tiene en cuenta solamente ángulos pequeños y no hay aproximación sobre la altura del sistema. Como lo indica la flecha a la izquierda se han ordenado los estados por sus números cuánticos que corresponden a $(n, m, l)$ y $(m, i, j)$ para los sistemas piramidal y cónico respectivamente.

Figura 11. Densidad de probabilidad de los primeros 10 estados para el sistema piramidal, la figura de arriba hacia abajo corresponde a las proyecciones en los planos $x y, x z$ y $y z$ respectivamente. La figura se ha realizado con $h=15 \mathrm{~nm}$ y $\theta=30^{\circ}$, los planos se han tomado en el centro de gravedad de la estructura.

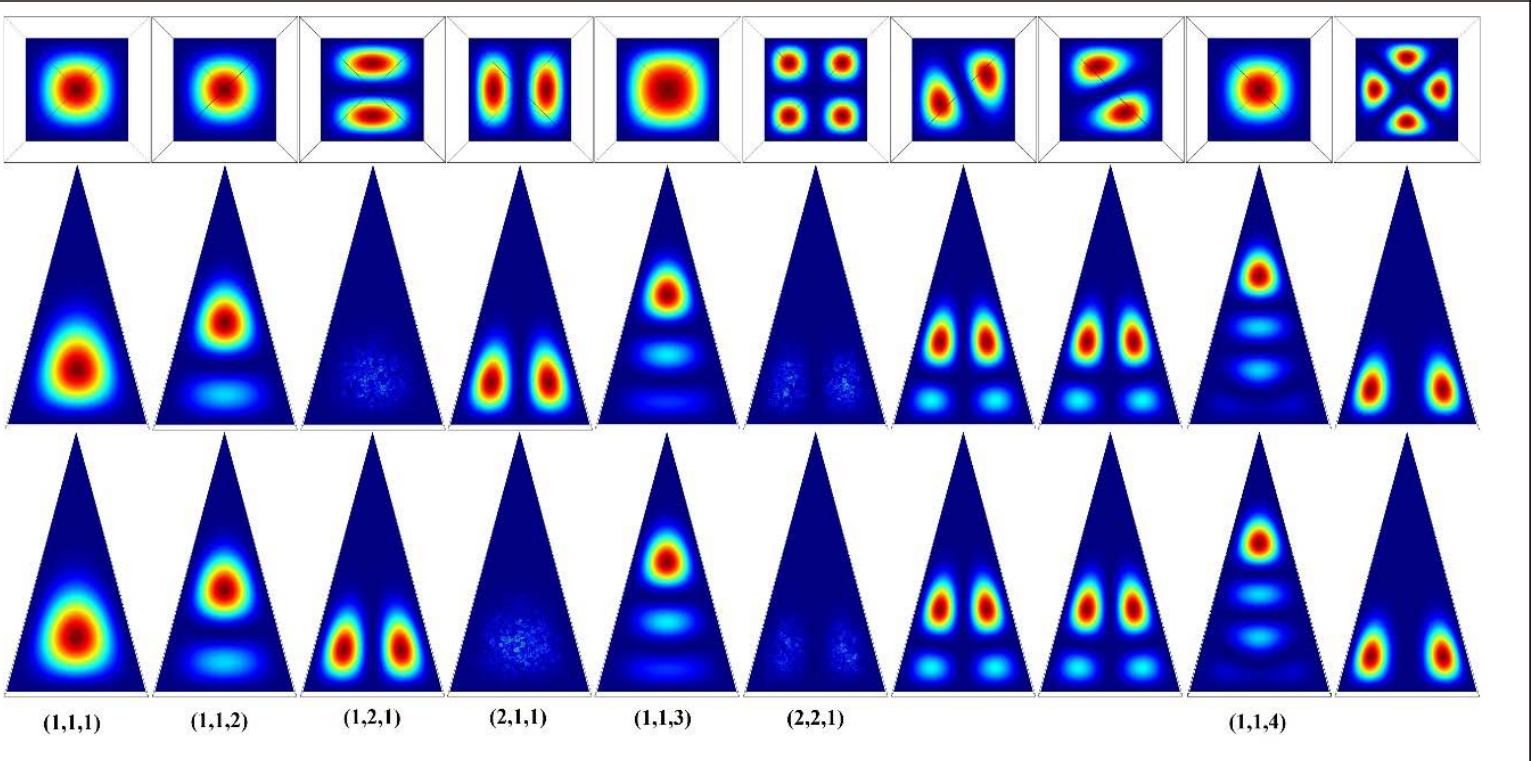


Figura 12. Densidad de probabilidad de los primeros 10 estados para el sistema cónico, la figura de arriba hacia abajo corresponde a las proyecciones en los planos $x y$, $x z$ y $y z$ respectivamente. La figura se ha realizado con $h=15 \mathrm{~nm}$ y $\theta=30^{\circ}$, los planos se han tomado en el centro de gravedad de la estructura.

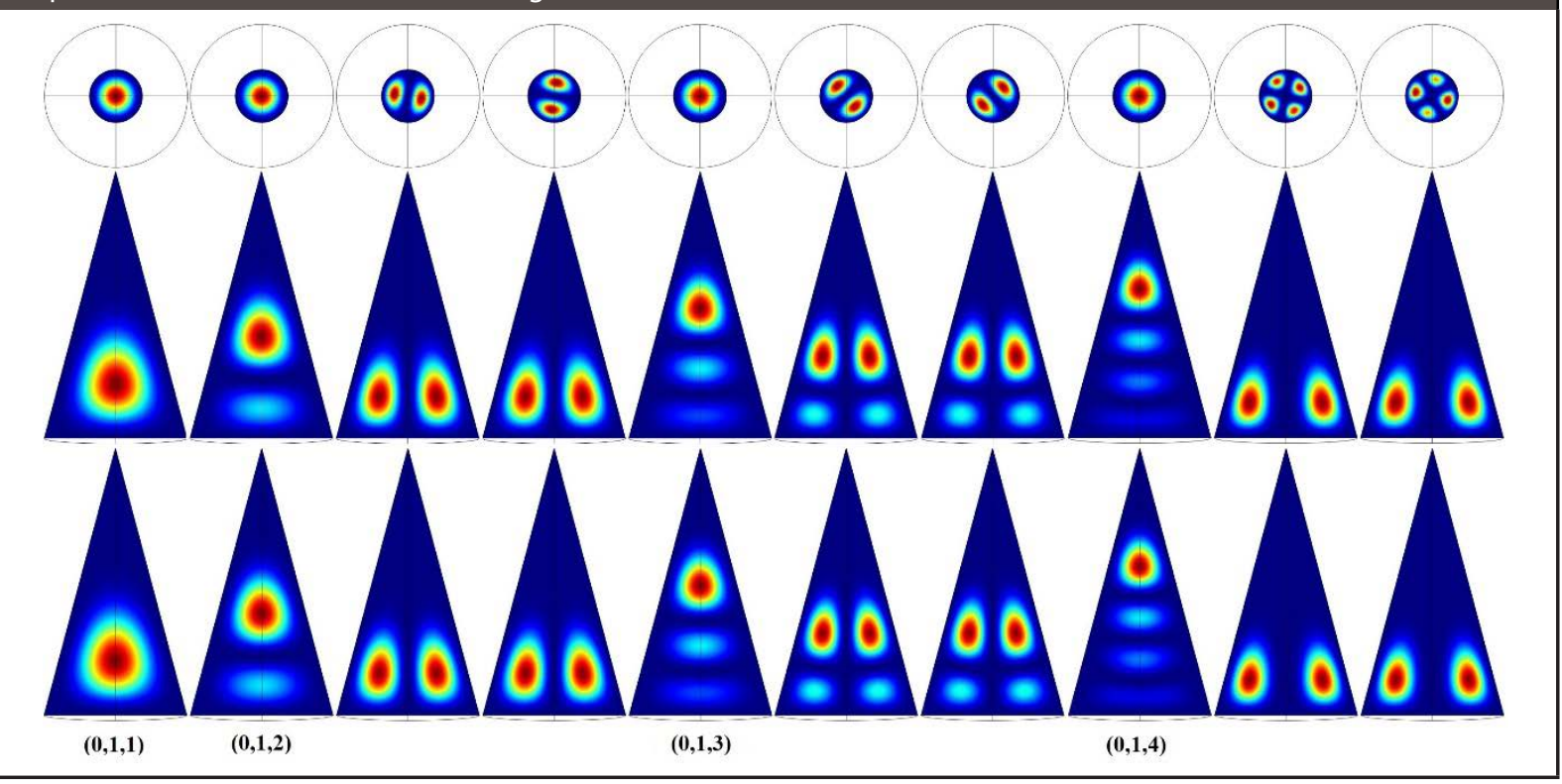

En las Figuras 11 y 12 se observa la densidad de probabilidad electrónica dada por $|\Phi(x, y, z)|^{2}$ para el sistema piramidal y cónico respectivamente. Se han indicado los números cuánticos que determinan cada uno de los estados que están de acuerdo según las Ecuaciones (8) y (16) para el sistema piramidal y cónico con las variables $(n, m, l)$ y $(m, i, j)$ respectivamente. Los estados cuyos números cuánticos no se presentan corresponden a estados que no tienen una dirección definida $x$ o $y$, estos estados presentan una rotación, por ejemplo, para el sistema cónico, el segundo y tercer estado excitado son estados tipo $p$ que presentan una rotación alrededor del eje $z$. Para los resultados presentados se ha usado la configuración $h=15 \mathrm{~nm}$ y $\theta=30^{\circ}$. La fila superior en ambas figuras corresponde a la proyección en el plano $x y$ donde se observa el sistema desde la parte superior, el plano observado está localizado en el centro de gravedad de la estructura, tanto la pirámide como el cono, esta es la razón por la cual el plano no llena completamente la forma piramidal y cónica, la fila intermedia y la fila inferior corresponden a las proyecciones en los planos $x z$ y $y z$ respectivamente. Las columnas de izquierda a derecha corresponden a distintos estados del sistema, estando el estado fundamental a la izquierda y los estados más altos más hacia la derecha. Claramente el estado fundamental para ambas estructuras se extiende sobre cada volumen tratando de llenarlo completamente y manteniendo la simetría propia tanto para la pirámide como para el cono. El segundo y tercer estado excitado para ambos sistemas presentan degeneración, lo cual puede verse con facilidad observando la distribución espacial que se da en la densidad de probabilidad para ambos estados. Lo mismo ocurre con los estados sexto y séptimo excitados para el sistema piramidal. Hay que resaltar que debido a que el punto cuántico cónico satisface un mayor número de grupos de simetría, se presenta un mayor número de degeneraciones por simetría en este sistema comparándolo con el sistema piramidal.

\section{Conclusiones}

En este artículo se ha realizado una comparación entre los resultados cuasianalíticos obtenidos considerando ángulos pequeños para los puntos cuánticos piramidal y cónico de GaAs y los resultados 
obtenidos por el método más preciso de elementos finitos. Los estados electrónicos confinados, así como las funciones de onda correspondientes se han obtenido por ambos procedimientos. Las Figuras 8, 9 y $\mathbf{1 0}$ corroboran que la aproximación cuasianalítica en efecto es una aproximación suficientemente válida cuando se está interesado en realizar un estudio de estos sistemas para ángulos no muy grandes (no superiores a $90^{\circ}$ ).

\section{Agradecimientos}

Esta investigación fue parcialmente respaldada por las agencias colombianas: CODI-Universidad de Antioquia (Estrategia de Sostenibilidad de la Universidad de Antioquia y proyectos: "Efectos ópticos intersubbanda, no lineales de segundo orden y dispersión Raman, en sistemas asimétricos de pozos cuánticos acoplados" y "Efectos de capas delta dopadas en pozos cuánticos como fotodetectores en el infrarrojo") y la Facultad de Ciencias Exactas y Naturales-Universidad de Antioquia (CAD-proyecto de dedicación exclusive 2017-2018 y ALM-proyecto de dedicación exclusive 2018-2019).

\section{Referencias}

Andrade C. G, Cabral Filho P. E., Tenório D. PL, Santos B. S., Beltrao E. IC, Fontes A., Carvalho L. B. (2013). Evaluation of Glycophenotype in Breast Cancer by Quantum Dot-lectin Histochemistry. Int. J. Nanomed. 8, pp 4623 - 4629.

Bahramiyan H. (2018). Electric field y impurity effect on nonlinear optical rectification of a double cone like quantum dot. Opt. Mater 75, pp 187 - 195.

Baier M. H., C. Constantin, Pelucchi E., y Kapon E. (2004). Electroluminescence from a single pyramidal quantum dot in a light-emitting diode. Appl. Phys. Lett.84, pp 1967 - 1969.

Bailey R. E., Smith A. M. y Shuming N. (2004). Quantum dots in biology and medicine. Physica E 25, pp 1 - 12.

COMSOL Multiphysics, v. 5.2a. COMSOL AB, Stockholm, Sweden.

Cunha C.R.A., Oliveira A.D.P.R., Firmino T.V.C., Tenório D.P.L.A., Pereira G., Carvalho L.B., Santos B.S., Correia
M.T.S., Fontes A. (2018). Biomedical Applications fo Glyconanoparticles Based on Quantum Dots, Biochim. Biophys. Acta, 1862, pp 427 - 439.

Duque C.A., Gil-Corrales A., Morales A.L., Restrepo R.L., Mora-Ramos M.E. y Monsalve-Calderón K. (2017). Electron Raman Scattering and Raman Gain in Pyramidal Semiconductor Quantum Dots, J. Nanosci. Nanotechno. 17, pp 1140-1148.

Duque C.A., Gil-Corrales A., Morales A.L., Restrepo R.L., Mora-Ramos M.E. (2017). Donor-impurity-related optical response and electron Raman scattering in GaAs cone-like quantum dots, Physica B 507, pp 76-83.

Hayrapetyan D. B., Kazaryan E. M. y Sarkisyan H. A. (2016). Magneto-absorption in conical quantum dot ensemble: Possible applications for QD LED. Opt. Commun. 371, pp 138 - 143.

Huggenberger A., Schneider C., Drescher C., Heckelmann S., Heindel T., Reitzenstein S., Kamp M., Hofling S., Worschech L. y Forchel A. (2011). Site-controlled In(Ga)As/GaAs quantum dots for integration into optically and electrically operated devices J. Cryst. Grown 323, pp 194 - 197.

Jadupati Nag, Rawat K., Asokan K., Kanjilal D., Bohidar H.B. (2018). Zener diode behavior of nitrogen-doped graphene quantum dots. Physica E 17, pp 13181 13200.

Jarlov C., Gallo P., Calic M., Dwir B., Rudra A. (2012). Bound and anti-bound biexciton in site-controlled pyramidal GaInAs/GaAs quantum dots. Appl. Phys. Lett. 101, pp 191101-1 191101-4.

Khordad R. y Bahramiyan H. (2014). Optical Properties of a GaAs Cone-Like Quantum Dot: Second and Third Harmonic Generation. Opt. Spectrosc. 117, pp 447452.

Khordad R., Bahramiyan H. y Mohammadi S.A. (2016). Influence of impurity on binding energy and optical properties of lens shaped quantum dots: Finite element method and Arnoldi algorithm. Chinese J. Phys. 54, pp $20-32$.

Lozovski V. y Piatnytsia V. (2011). The Analytical Study of Electronic and Optical Properties of Pyramid-Like and Cone-Like Quantum Dots. J. Comput. Theor. Nanos. 8, pp 1-9.

Luhluh K. J., Boda A., Shankar I. V., Raju Ch. N. y Chatterjee A. (2018). Magnetic field effect on the energy levels of an exciton in a GaAs quantum dot: Application for excitonic lasers. Sci. Rep-Uk 8, pp 5073 - 5086. 
Manoj K. M., Hofsass H. C. y Vetter U. (2016). Photon-Upconverting Materials: Advances and Prospects for Various Emerging Applications. Intech., 298, pp 109 $-131$.

Norris D. J. y Bawendi M. G. (1996). Measurement and assignment of the size-dependent optical spectrum in CdSe quantum dots. Phys. Rev. B, 53, pp 16338 16346.

Pickering S., Kshirsagar A., Ruzyllo J., and Xu J. (2012). Patterned mist deposition of tri-colour CdSe/ZnS quantum dot films toward RGB LED devices. OptoElectron Rev. 20, pp 148 - 152.

Ponnusamy B., Sharmistha S., y Avadhesha S. (2007). Sugar-Quantum Dot Conjugates for a Selective and Sensitive Detection of Lectins. Bioconjugate Chem. 18, pp $146-151$.

Safeera T.A., Khanal R., Medvedeva J. E., Martinez A. I., Vinitha G., Anila E.I. (2018). Low temperature synthesis and characterization of zinc gallate quantum dots for optoelectronic applications. J. Alloy Compd. 740, pp 567 - 589.

Sagadevan S. y Dakshanamoorthy A. (2012). Nanomaterials for Nonlinear Optical (NLO) Applications: A Review. Rev. Adv. Mater. Sci. 30, pp 243-253.

Yamaguchi M., Asano T., y Noda S. (2008). Photon emission by nanocavity-enhanced quantum anti-Zeno effect in solid-state cavity quantum-electrodynamics. Opt. Express. 16, pp 18067 - 18081.

Zrazhevskiy P. y Xiaohu G. (2009). Multifunctional quantum dots for personalized medicine. Nano Today. 4 , pp $414-428$.

\section{PARA CITAR ESTE ARTÍCULO / TO REFERENCE THIS ARTICLE / PARA CITAR ESTE ARTIGO /}

Gil-Corrales, J.A.; Morales-Aramburo, A.L.: Duque-Echeverri, C.A. (2018). Estados electrónicos de puntos cuánticos piramidales y cónicos. Revista EIA, 15(30), julio-diciembre, pp. 161175. [Online]. Disponible en: https://doi.org/10.24050/ reia.v15i30.1257 